\title{
Corrected Hawking temperature of acoustic rotating black hole
}

\author{
Chandra Rekha Mahanta ${ }^{1}$, Rajesh Misra ${ }^{2}$ \\ ${ }^{1}$ Department of Mathematics, Cotton College, Guwahati-781001, India \\ ${ }^{2}$ Department of Mathematics, Gauhati University, Guwahati-781014, India \\ *Corresponding author E-mail:crmahanta4@gmail.com
}

\begin{abstract}
In this paper, the corrected Hawking temperature of $(2+1)$ dimensional acoustic rotating black hole has been calculated by using tunneling method. For this purpose, the r-t sector of the metric is isolated from the angular part by taking a transformation of the time and the azimuthal angle co-ordinates in the exterior region of the event horizon. The massless particle of this black hole obeys the Klein- Gordon equation of motion.
\end{abstract}

Keywords: Black Hole; Classical Theories of Gravity; Hawking Temperature; Hawking Radiation; Tunneling Method.

\section{Introduction}

The topic of black hole thermodynamics has been a subject of great interest since the 1970's when Bekenstein (1973) first conjectured that there is a fundamental relationship between the properties of black holes and the laws of thermodynamics. Stephen Hawking strengthened this conjecture by showing that black holes can emit any kind of particles via thermal radiation, known as Hawking radiation, when quantum effects are taken into account (Hawking1975, Gibbons \& Hawking 1977a, 1977b). It is shown that there is small but a finite probability for any kind of particles to tunnel through the barrier of the quantum vacuum and escape the black hole horizons as a real particle with positive energy, leaving the particles with negative energy inside the horizon of the black hole. Hawking radiation implies that black hole could slowly evaporate emitting quantas and the rate of particle emission is as if the black hole were a hot body of temperature proportional to its surface gravity. This remarkable discovery leads to physical information about the classically forbidden region inside the horizon. Recently, a semi-classical method of controlling Hawking radiation as a tunneling effect has been developed (Kraus \& Wilczek 1995a, 1995b, Srinivasan \& Padmanavan 1999, Parikh \& Wilczek 2000, Shankaranarayanan et al. 2001, 2002, Kerner \& Mann 2006, Banerjee \& Modak 2009a, Mirza \& Sherkatghanad 2011). This is a particularly interesting method for calculating black hole temperature since it provides a dynamical model of the black hole radiation. In the tunneling approach, the particles are allowed to follow classically forbidden trajectories in a coordinate system which is well behaved at the horizon. This method in volves calculating the imaginary part of the action for the classically forbidden process of s-wave emission across the horizon, which in turn related to the Boltzmann factor for emission at the Hawking temperature. Using the WKB approximation, the tunneling probability for the classically forbidden trajectory of the swave coming from inside to outside the event horizon is given

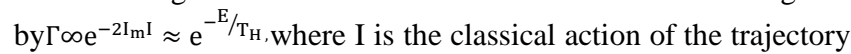
and $\mathrm{E}$ is the energy of the tunneling particles. To calculate the imaginary part of the action for the emitted particles, the complex path method, also known as Hamilton- Jacobi method is used frequently. This method was first proposed by K. Srinivasan \& T. Padmanavan (1999) and subsequently developed by many authors. The Hamilton-Jacobi methods involve consideration of an emitted scalar particle, ignoring its self-gravitation and assume that its action satisfies the relativistic Hamilton- Jacobi equation. The method is motivated by applying the WKB approximation to the KleinGordon equation. Related research has shown that the black hole tunneling method can be successfully applied to variety of dynamical black holes. This method has acquired a growing interest and has been used by many authors to find out the Hawking temperature for different black holes and for more complicated spacetimes. Recently, Shankarnarayanan et al. (2002) obtained the corrected temperature associated with the Hawking radiation in different coordinate systems by applying the Hamilton-Jacobi method for the Schwarzschild space time. Angheben et al. (2005) applied Hamilton-Jacobi equation and WKB approximation to deal with the tunneling for extremal and rotating black hole. Criscienzo et al. (2007) analyzed the instability against emission of massless particles by the trapping horizon of an evolving black hole with the use of the Hamilton-Jacobi method. Jiang et al. (2009) performed an analysis of Hawking radiation about apparent horizon in a FRW universe in which the Hawking radiation of a black hole is treated as the quantum tunneling by Hamilton-Jacobi method beyond semi-classical approximation. Considering the contribution of both the outer and inner horizons Bo and Wen-Biao (2010) have applied the Hamilton-Jacobi method to a Kerr-Newman black hole and obtained a negative temperature of the inner horizon. Mirza and Sherkatghanad (2011) studied the AdS rotating black hole for the BHT massive gravity in three dimensions. They have calculated the corrected entropy of the rotating black hole solution of the new massive gravity using tunneling method and Cardy formula. Mahanta and Misra (2013a, 2013b) have calculated the corrected Hawking temperature for the Warped $\mathrm{AdS}_{3}$ rotating black hole and $(2+1)$ dimensional BTZ rotating black hole by using tunneling method. Several other aspects of this method have also been discussed extensively by many authors (Arzano et al. 
2005, Medved \& Vagenas 2005, Akhmedov et al. 2006, Modak 2009, Banerjee \& Modak 2009b).

Although several decades have passed, the phenomenon of Hawking radiation is yet to be observed experimentally compared with the difficulties on the astrophysical side. Recently analog models of general relativity have received great attention as these models are shedding light on possible experimental verifications of some fundamental problems in black hole physics such as the evaporation of black hole and semi-classical quantities. Unruh (1981) first proposed the idea of using acoustic geometry on the use of supersonic acoustic flows as an analogy to gravitating systems. The basis of the analogy between gravitational black hole and acoustic black hole comes from the possibility of experimental observation of the acoustic analog of Hawking radiation from regions of flow of inviscid and barotropic fluids behaving as outer trapped surfaces viz. acoustic event horizons. As discussed by Unruh (1995) and subsequently by many authors, an acoustic event horizon will emit Hawking radiation in the form of a thermal bath of phonons at a temperature $\mathrm{T}_{\mathrm{H}}=\frac{\hbar \mathrm{g}_{\mathrm{H}}}{2 \pi \mathrm{C}}, \mathrm{g}_{\mathrm{H}}$ being the normalizer to have the dimensions of a physical acceleration. The equation of motion for the acoustic disturbance is identical to the Klein- Gordon equation for a massless scalar field minimally coupled to gravity in a curved space time (Visser 1998). Visser showed how to define the ergosphere, trapped regions, acoustic apparent horizon and acoustic event horizon for a supersonic fluid flow with a sink at the origin. With Visser's works (Visser 1993, 1998, 1999, Novello et al. 2002, Barcelo et al. 2005) acoustic black hole has received an exponentially growing attention in the field of research. Many black hole issues have already been treated as field theoretical problems in fluid because the acoustic analogue of a rotating black hole is found to be useful in studying various aspects of black hole physics. Berti et al. (2004) computed the reflection coefficients of the super radiant scattering displayed by the ergoregion of $(2+1)$ dimensional acoustic black hole. Lepe and Saavedra (2005) presented an exact expression for the quasinormal modes of acoustic disturbances in a rotating $(2+1)$ dimensional sonic black hole (draining bathtub fluid flow) in the low frequency limit. Berti (2005) have evaluated the classical wave propagation in a rotating acoustic $(2+1)$ dimensional black hole. Kim et al. (2006) studied the "draining bathtub" as an acoustic analogue of a threedimensional rotating black hole. Bercelo et al. (2006) discussed the issue of quasi-particle production by analogue black holes with particular attention to the possibility of reproducing Hawking radiation in a laboratory. Carusotto et al. (2008) reported numerical evidence of Hawking emission of Bogoliubov phonons from a sonic horizon in a flowing one-dimensional atomic Bose-Einstein condensate. Kim et al. (2008) studied thermodynamic quantities of an acoustic black hole and its thermodynamic stability in a cavity based on the generalized uncertainty principle and found that the acoustic black hole can decay into the remnant or the large black hole. Recently Lombardo et al. (2012) studied the process of decoherence in acoustic black holes. Lemos (2013) considered the (2+1)-dimensional draining bathtub metric and studied its quasi normal modes, its superresonance features, its instabilities when surrounding it by a mirror and the possibility of turning it into a sonic bomb or a power plant. Many fluid systems have also been investigated on a variety of analogue models of acoustic black holes, including gravity wave, electromagnetic wave etc. In the context of what has been stated above, it will be logical and relevant to calculate the corrected Hawking temperature of acoustic rotating black hole by tunneling method via Hamilton-Jacobi ansatz. For this purpose, however, we have to isolate the r-t sector of the metric from the angular rotating part by taking a suitable transformation of co-ordinates.

In our work, we have calculated the corrected Hawking temperature of $(2+1)$ dimensional acoustic rotating black hole by tunneling method. The paper is organized as follows. In Sec. 2 we consider the $(2+1)$ dimensional acoustic rotating black hole model. In Sec. 3 the correction to the semi-classical Hawking temperature is obtained for this metric. We conclude the paper with a brief discussion in Sec. 4.

\section{2. (2+1) Dimensional acoustic rotating black hole model}

For the $(2+1)$ dimensional acoustic rotating black hole, we consider the acoustic metric of the so called 'draining bathtub' fluid flow with a sink at the origin first introduced by Visser (1998)

$d s^{2}=-\left(C^{2}-\frac{A^{2}+B^{2}}{r^{2}}\right) d t^{2}-\frac{2 A}{r} d r d t-$

$2 \mathrm{Bd} \varphi \mathrm{dt}+\mathrm{dr}^{2}+\mathrm{r}^{2} \mathrm{~d} \varphi^{2}$

Where $\mathrm{t}$ is the time coordinate. The acoustic horizon is located at

$\mathrm{r}_{\mathrm{H}}=\frac{\mathrm{A}}{\mathrm{C}}$

And the ergosphere forms at

$r_{E}=\frac{\sqrt{A^{2}+B^{2}}}{C}$

WhereC is the speed of sound?

Writing the metric (1) in the form

$\mathrm{ds}^{2}=-\mathrm{C}^{2} \mathrm{dt}^{2}+\left(\mathrm{dr}-\frac{\mathrm{A}}{\mathrm{r}} \mathrm{dt}\right)^{2}+\left(\mathrm{rd} \varphi-\frac{\mathrm{B}}{\mathrm{r}} \mathrm{dt}\right)^{2}$

And then taking the transformations of the time and the azimuthal angle coordinates in the exterior region of $\mathrm{A} / \mathrm{C}<r<\propto$ (Basak and Majumdar 2003), namely

$\mathrm{dt} \rightarrow \mathrm{dt}+\frac{\mathrm{Ar}}{\mathrm{r}^{2} \mathrm{C}^{2}-\mathrm{A}^{2}} \mathrm{dr} \mathrm{d} \varphi \rightarrow \mathrm{d} \varphi+\frac{\mathrm{AB}}{\mathrm{r}\left(\mathrm{r}^{2} \mathrm{C}^{2}-\mathrm{A}^{2}\right)} \mathrm{dr}$

The metric (2) can be rewritten in the form

$\mathrm{ds}^{2}=-\mathrm{N}^{2}(\mathrm{r}) \mathrm{dt}^{2}+\frac{1}{\mathrm{~N}^{2}(\mathrm{r})} \mathrm{dr}^{2}+\mathrm{r}^{2}\left(\mathrm{~d} \varphi-\Omega_{0} \mathrm{dt}\right)^{2}$

Where

$\mathrm{N}^{2}(\mathrm{r})=1-\frac{\mathrm{A}^{2}}{\mathrm{C}^{2} \mathrm{r}^{2}}=\frac{\mathrm{r}^{2}-\mathrm{r}_{\mathrm{H}}^{2}}{\mathrm{r}^{2}}$

And

$\Omega_{0}(\mathrm{r})=\frac{\mathrm{B}}{\mathrm{Cr}^{2}}=\Omega_{\mathrm{H}} \frac{\mathrm{r}_{\mathrm{H}}^{2}}{\mathrm{r}^{2}}$

The metric (3) looks similar to that of the rotating BTZ black hole (Banados et al. 1992), but it has a difference with the lapse function $\mathrm{N}(\mathrm{r})$.

$\operatorname{HereN}^{2}(r)=1-\frac{A^{2}}{C^{2} r^{2}}=\frac{r^{2}-r_{H}^{2}}{r^{2}}$

Whereas

$\mathrm{N}_{\mathrm{BTZ}}^{2}(\mathrm{r})=\frac{\left(\mathrm{r}^{2}-\mathrm{r}_{+}^{2}\right)\left(\mathrm{r}^{2}-\mathrm{r}_{-}^{2}\right)}{\mathrm{r}^{2} \mathrm{l}^{2}}$

Where $\mathrm{l}^{2}=\frac{-1}{\Lambda}$

Moreover the acoustic black hole is asymptotically flat while the BTZ black hole has asymptotically anti-de-sitter space time.

The massless particle of the equation (3) obeys the Klein- Gordon equation of motion

$-\frac{\hbar^{2}}{\sqrt{-g}} \partial_{\mu}\left[g^{\mu \nu} \sqrt{-g} \partial_{\nu} \varphi\right]=0$ 


\section{Correction to the semi-classical Hawking temperature}

To solve the equation of motion (4) with the help of background metric (3), we can write the standard WKB (Wenzel-KramersBrillouin) ansatz for $\varphi$ as

$$
\varphi(r, t)=\exp \left[-\frac{i}{\hbar} s(r, t)\right]
$$

Putting the value of $\varphi$ from the equation (5) we can calculate (4) as,

$$
\begin{aligned}
& -\frac{\hbar}{\sqrt{-g}} \frac{\partial}{\partial \mu}\left[g^{\mu \nu} \sqrt{-g} \frac{\partial}{\partial v} \mathrm{e}^{-\frac{\mathrm{i}}{\hbar} \mathrm{S}}\right]=0 \\
& \Rightarrow \frac{\partial}{\partial r}\left[g^{r r} \sqrt{-g} \frac{\partial}{\partial r} e^{-\frac{i}{\hbar} s}\right]+\frac{\partial}{\partial t}\left[g^{t t} \sqrt{-g} \frac{\partial}{\partial t} e^{-\frac{i}{\hbar} s}\right]=0 \\
& \Rightarrow \frac{\partial}{\partial r}\left[-N^{2}(r) \frac{\partial}{\partial r} e^{-\frac{i}{\hbar} S}\right]+\frac{\partial}{\partial t}\left[\frac{1}{N^{2}(r)} \frac{\partial}{\partial t} e^{-\frac{i}{\hbar} s}\right]=0 \\
& \Rightarrow \frac{\partial}{\partial r}\left[-N^{2}(r)\left(\frac{-i}{\hbar}\right) e^{-\frac{i}{\hbar} S} \frac{\partial S}{\partial r}\right]+\frac{\partial}{\partial t}\left[\frac{1}{N^{2}(r)}\left(\frac{-i}{\hbar}\right) e^{-\frac{i}{\hbar} S} \frac{\partial S}{\partial t}\right]=0 \\
& \Rightarrow-\mathrm{N}^{2}(\mathrm{r})\left(\frac{-\mathrm{i}}{\hbar}\right) \mathrm{e}^{-\frac{\mathrm{i}}{\mathrm{h}} \mathrm{S}}\left(\frac{\partial \mathrm{S}}{\partial \mathrm{r}}\right)^{2}+\mathrm{N}^{2}(\mathrm{r}) \mathrm{e}^{-\frac{\mathrm{i}}{\mathrm{h}} \mathrm{S}} \frac{\partial^{2} \mathrm{~S}}{\partial \mathrm{r}^{2}}+\frac{\partial}{\partial \mathrm{r}}\left(\mathrm{N}^{2}(\mathrm{r})\right) \mathrm{e}^{-\frac{\mathrm{i}}{\mathrm{h}} \mathrm{S}} \frac{\partial \mathrm{S}}{\partial \mathrm{r}} \\
& \begin{array}{l}
+\frac{1}{\mathrm{~N}^{2}(\mathrm{r})}\left(\frac{-\mathrm{i}}{\hbar}\right) \mathrm{e}^{-\frac{\mathrm{i}}{\hbar} \mathrm{S}}\left(\frac{\partial \mathrm{S}}{\partial \mathrm{t}}\right)^{2}+\frac{1}{\mathrm{~N}^{2}(\mathrm{r})} \mathrm{e}^{-\frac{\mathrm{i}}{\hbar} \mathrm{S}} \frac{\partial^{2} \mathrm{~S}}{\partial \mathrm{t}^{2}} \\
=0
\end{array}
\end{aligned}
$$$$
\begin{aligned}
\Rightarrow-\mathrm{N}^{2}(\mathrm{r})\left(\frac{-\mathrm{i}}{\hbar}\right)\left(\frac{\partial \mathrm{S}}{\partial \mathrm{r}}\right)^{2}-\mathrm{N}^{2}(\mathrm{r}) \frac{\partial^{2} \mathrm{~S}}{\partial \mathrm{r}^{2}}-\frac{\partial}{\partial \mathrm{r}}\left(\mathrm{N}^{2}(\mathrm{r})\right) \frac{\partial \mathrm{S}}{\partial \mathrm{r}} \\
+\frac{1}{\mathrm{~N}^{2}(\mathrm{r})}\left(\frac{-\mathrm{i}}{\hbar}\right)\left(\frac{\partial \mathrm{S}}{\partial \mathrm{t}}\right)^{2}+\frac{1}{\mathrm{~N}^{2}(\mathrm{r})} \frac{\partial^{2} \mathrm{~S}}{\partial \mathrm{t}^{2}}=0
\end{aligned}
$$$$
\Rightarrow \frac{1}{\mathrm{~N}^{2}(\mathrm{r})}\left(\frac{\partial \mathrm{S}}{\partial \mathrm{t}}\right)^{2}-\mathrm{N}^{2}(\mathrm{r})\left(\frac{\partial \mathrm{S}}{\partial \mathrm{r}}\right)^{2}-\frac{\hbar}{\mathrm{i}} \frac{1}{\mathrm{~N}^{2}(\mathrm{r})} \frac{\partial^{2} \mathrm{~S}}{\partial \mathrm{t}^{2}}+\frac{\hbar}{\mathrm{i}} \mathrm{N}^{2}(\mathrm{r}) \frac{\partial^{2} \mathrm{~S}}{\partial \mathrm{r}^{2}}+
$$$$
\frac{\hbar}{\mathrm{i}} \frac{\partial}{\partial \mathrm{r}} \mathrm{N}^{2}(\mathrm{r}) \frac{\partial \mathrm{S}}{\partial \mathrm{r}}=0
$$

Here $S(r, t)$ can be expanded in the power of $\hbar$ as

$$
S(r, t)=S_{0}(r, t)+\sum_{i} \hbar^{i} S_{i}(r, t)
$$

For $\mathrm{i}=1,(7)$ becomes

$$
S=S_{0}+\hbar^{0} S_{0}+\hbar^{1} S_{1}
$$

Putting (8) in (6) we get,

$$
\begin{aligned}
& \frac{1}{N^{2}(r)}\left(\frac{\partial}{\partial t}\left(S_{0}+\hbar^{0} S_{0}+\hbar^{1} S_{1}\right)\right)^{2}-N^{2}(r)\left(\frac { \partial } { \partial r } \left(S_{0}+\hbar^{0} S_{0}+\right.\right. \\
& \left.\left.\hbar^{1} S_{1}\right)\right)^{2}-\frac{\hbar}{i} \frac{1}{N^{2}(r)} \frac{\partial^{2}}{\partial t^{2}}\left(S_{0}+\hbar^{0} S_{0}+\hbar^{1} S_{1}\right)+\frac{\hbar}{i} N^{2}(r) \frac{\partial^{2}}{\partial r^{2}}\left(S_{0}+\right. \\
& \left.\hbar^{0} S_{0}+\hbar^{1} S_{1}\right)+\frac{\hbar}{i} \frac{\partial}{\partial r} N^{2}(r) \frac{\partial}{\partial r}\left(S_{0}+\hbar^{0} S_{0}+\hbar^{1} S_{1}\right)=0
\end{aligned}
$$

Neglecting the coefficient of $\hbar$ we get,

$$
\begin{gathered}
-N^{2}(r)\left[\frac{\partial S_{0}}{\partial r}+\hbar^{0} \frac{\partial S_{0}}{\partial r}+\hbar^{1} \frac{\partial S_{1}}{\partial r}\right]^{2} \\
+\frac{1}{N^{2}(r)}\left[\frac{\partial S_{0}}{\partial t}+\hbar^{0} \frac{\partial S_{0}}{\partial t}+\hbar^{1} \frac{\partial S_{1}}{\partial t}\right]^{2}=0 \\
\begin{aligned}
\Rightarrow\left(N^{2}(r)\right)^{2}\left[\frac{\partial S_{0}}{\partial r}+\hbar^{0} \frac{\partial S_{0}}{\partial r}+\hbar^{1} \frac{\partial S_{1}}{\partial r}\right]^{2} \\
=\left[\frac{\partial S_{0}}{\partial t}+\hbar^{0} \frac{\partial S_{0}}{\partial t}+\hbar^{1} \frac{\partial S_{1}}{\partial t}\right]^{2}
\end{aligned}
\end{gathered}
$$

$$
\Rightarrow \frac{\partial S_{0}}{\partial t}+\hbar^{0} \frac{\partial S_{0}}{\partial t}+\hbar^{1} \frac{\partial S_{1}}{\partial t}= \pm N^{2}(r)\left(\frac{\partial S_{0}}{\partial r}+\hbar^{0} \frac{\partial S_{0}}{\partial r}+\hbar^{1} \frac{\partial S_{1}}{\partial r}\right)
$$

Equating the coefficient of $\hbar^{0}, \hbar^{1}$ respectively we get,

$\frac{\partial S_{0}}{\partial t}= \pm N^{2}(r) \frac{\partial S_{0}}{\partial r}$

$\frac{\partial S_{1}}{\partial t}= \pm N^{2}(r) \frac{\partial S_{1}}{\partial r}$

Similarly,

$\frac{\partial S_{2}}{\partial t}= \pm N^{2}(r) \frac{\partial S_{2}}{\partial r}$

And so on.

All the equations obtained above are identical and so their solutions are also related to each other. We assume that any $S_{i}(r, t)$ can differ from $S_{0}(r, t)$ by a proportionality factorso that we may write

$S(r, t)=\left(1+\sum_{i} \hbar^{i} \gamma_{i}\right) S_{0}(r, t)$

The dimension of $\gamma_{i}$ is equal to the dimension of $\hbar^{-i}$. Thus the $\gamma_{\mathrm{i}}$ 'scan be expressed in terms of the dimensionless constant. In $(2+1)$ dimensions we can choose $G=C=\kappa_{\beta}=\frac{1}{4 \pi \epsilon_{0}}=1$ and $\sqrt{\hbar}$ can be replaced by plank length $\left(l_{p}\right)$. The length parameters for these black holes is $r_{H}$. So we have,

$S(r, t)=\left(1+\sum_{i} \frac{\beta_{i} \hbar^{i}}{H_{a c}^{i}}\right) S_{0}(r, t)$

Where $\beta_{i}$ 's are dimensionless constantsand $H_{a c}=a_{1} r_{H}^{2}, a_{1}$ being an arbitrary constant (Banerjee and Modak 2009b). Isolating the semi classical action for the $r-t$ sector near the horizon, we obtain

$S_{0}(r, t)=\omega t+\overline{S_{0}}(r)$

Where the total energy of the tunneling particle near the horizon approximation is given by

$\omega=E-J \Omega_{+}$

Now from (10.1) and (13) we get,

$\frac{\partial}{\partial t}\left[\omega t+\overline{S_{0}}(r)\right]= \pm N^{2}(r) \frac{\partial}{\partial r}\left[\omega t+\overline{S_{0}}(r)\right]$

$\Rightarrow \omega= \pm N^{2}(r) \frac{\partial \overline{S_{0}}}{\partial r}$

$\Rightarrow \frac{\partial \overline{S_{0}}}{\partial r}= \pm \frac{\omega}{N^{2}(r)}$

$\Rightarrow \partial \overline{S_{0}}= \pm \frac{\omega}{N^{2}(r)} \partial r$

Integrating we get,

$\overline{S_{0}}= \pm \omega \int \frac{d r}{N^{2}(r)}$

Here $+(-)$ sign shows that the particle is ingoing (outgoing) the event horizon. As a result substituting $S_{0}(r, t)$ and $\overline{S_{0}}$ in (12) we get,

$S(r, t)=\left(1+\sum_{i} \frac{\beta_{i} \hbar^{i}}{H_{a c}^{i}}\right)\left(\omega t \pm \omega \int \frac{d r}{N^{2}(r)}\right)$

A solution for the scalar field in the presence of the higher order correction to the semi classical action is given by

$\varphi_{\text {in }}=\exp \left[-\frac{i}{\hbar}\left(1+\sum_{i} \frac{\beta_{i} \hbar^{i}}{H_{a c}^{i}}\right)\left(\omega t+\omega \int \frac{d r}{N^{2}(r)}\right)\right]$ 
$\varphi_{\text {out }}=\exp \left[-\frac{i}{\hbar}\left(1+\sum_{i} \frac{\beta_{i} \hbar^{i}}{H_{a c}^{i}}\right)\left(\omega t-\omega \int \frac{d r}{N^{2}(r)}\right)\right]$

Where ingoing and outgoing particles cross the event horizon on different paths.

Now, for the tunneling of a particle across the event horizon, the nature of the co-ordinates of $r$ and $t$ change. The time-like coordinate $t$ outside the event horizon changes to the space-time coordinate inside the event horizon and likewise for the outside space-time co-ordinate $r$. Since the metric coefficients for the r-t sector change sign at the two sides of the event horizon, the outgoing particle cannot cross the event horizon classically. Therefore, the path on which the tunneling takes place has an imaginary time coordinate $\left(I_{m} \mathrm{t}\right)$. We can thus write the ingoing and outgoing probabilities as,

$p_{\text {in }}=\left|\varphi_{\text {in }}\right|^{2}=\exp \left[\frac{2}{\hbar}\left(1+\sum_{i} \frac{\beta_{i} \hbar^{i}}{H_{a c}^{i}}\right)\left(\omega I_{m} t+\omega I_{m} \int \frac{d r}{N^{2}(r)}\right)\right]$

$p_{\text {out }}=\left|\varphi_{\text {out }}\right|^{2}=\exp \left[\frac{2}{\hbar}\left(1+\sum_{i} \frac{\beta_{i} \hbar^{i}}{H_{a c}^{i}}\right)\left(\omega I_{m} t-\omega I_{m} \int \frac{d r}{N^{2}(r)}\right)\right](20)$

In the classical limit, the ingoing particle probability is unity; therefore we have, from (19)

$I_{m} t=-I_{m} \int \frac{d r}{\mathrm{~N}^{2}(\mathrm{r})}$

Now, $\mathrm{p}_{\text {out }}$ can be expressed as,

$\mathrm{p}_{\text {out }}=\exp \left[\frac{-4}{\hbar} \omega\left(1+\sum_{\mathrm{i}} \frac{\beta_{\mathrm{i}} \hbar^{\mathrm{i}}}{\mathrm{H}_{\mathrm{ac}}^{\mathrm{i}}}\right) \mathrm{I}_{\mathrm{m}} \int \frac{\mathrm{dr}}{\mathrm{N}^{2}(\mathrm{r})}\right]$

Identifying the temperature of this black hole by using the Principle of "detailed balance" (Srinivasan and Padmanavan 1999; Shankaranarayanan et al. 2001, 2002) for the ingoing and outgoing probabilities

$\frac{\mathrm{p}_{\text {out }}}{\mathrm{p}_{\text {in }}}=\exp \left(-\frac{\omega}{\mathrm{T}}\right)$

Or

$\mathrm{p}_{\text {out }}=\exp \left(-\frac{\omega}{\mathrm{T}}\right)$

We obtain the corrected Hawking temperature as,

$\mathrm{T}=\mathrm{T}_{\mathrm{H}}\left(1+\sum_{\mathrm{i}} \frac{\beta_{\mathrm{i}} \mathrm{5}^{\mathrm{i}}}{\mathrm{H}_{\mathrm{ac}}^{\mathrm{i}}}\right)^{-1}$

Where, $\mathrm{T}_{\mathrm{H}}$ is the semi classical Hawking temperature and other terms are corrections to the higher order quantum effect.

\section{Conclusion}

In this paper, we have used tunneling method to calculate the corrected Hawking temperature for the $(2+1)$ dimensional acoustic rotating black hole. For this purpose $r-t$, sector is isolated through a transformation of the time and the azimuthal angle co-ordinates in the exterior region of the event horizon. The massless particle of this black hole obeys the Klein-Gordon equation of motion. Considering the ingoing particle probability to be unity, it is found that the corrected Hawking temperature contains the semiclassical Hawking temperature and a factor giving the corrections to the higher-order quantum effect.

\section{References}

[1] Bekenstein, J. D., (1973).Black holes and entropy. Physical Review D 7, P 2333-2346. http://dx.doi.org/10.1103/physrevd.7.2333.
[2] Hawking, S. W., (1975).Particle creation by black holes. Communications in Mathematical Physics 43, P 199- 200. http://dx.doi.org/10.1007/BF02345020.

[3] Gibbons, G. W., Hawking. S. W., (1977a).Cosmological Event Horizons, Thermodynamics and Particle Creation. Physical Review D 15 P 2738-2751. http://dx.doi.org/10.1103/physrevd.15.2738.

[4] Gibbons, G. W., Hawking. S. W., (1977b).Action Integrals and partition functions in Quantum Gravity. Physical Review D 15, P 2752 2756. http://dx.doi.org/10.1103/physrevd.15.2752.

[5] Kraus, P., Wilczek, F., (1995a). Self-Interaction correction to black hole radiation. Nuclear Physics B 433, P 403-420. http://dx.doi.org/10.1016/0550-3213(94)00411-7.

[6] Kraus, P., Wilczek, F., (1995b). Effect of self-interaction on charged black hole radiance. Nuclear Physics B 437, P 231-242. http://dx.doi.org/10.1016/0550-3213(94)00588-6.

[7] Srinivasan, K., Padmanavan, T., (1999). Particle production and complex path analysis. Physical Review D 60, P 024007-024032. http://dx.doi.org/10.1103/PhysRevD.60.024007.

[8] Parikh, M. K., Wilczek, F., (2000). Hawking radiation as tunneling. Physical Review Letters 85, p 5042-5045. http://dx.doi.org/10.1103/physrevlett.85.5042.

[9] Shankaranarayanan, S., Srinivasan, K., Padmanabhan, T., (2001). Method of complex paths and general covariance of hawking radiation. Modern Physics letters A 16, P 571-578. http://dx.doi.org/10.1142/S0217732301003632.

[10] Shankaranarayanan, S., Padmanabhan, T., Srinivasan, T., (2002). Hawking radiation in different coordinate settings: complex path approach. Classical and Quantum Gravity 19, P 2671-2688. [grqc/0010042] [SPIRES] http://dx.doi.org/10.1088/02649381/19/10/310.

[11]Kerner,R., Mann,R. B., (2006).Tunneling, Temperature and TuabNUT black holes.PhysicalReviewD 73, P 104010-104031.

[12]Banerjee R., Modak, S. K., (2009a). Quantum tunneling, Black body spectrum and non- logarithmic entropy corrected for lovelock black holes. Journal of High Energy Physics 11, 073.

[13] Mirza, B., Sherkatghanad,Z., (2011). Corrected entropy of the rotating black hole solution of the new massive gravity using the Tunneling method and Cardy formula. Physical ReviewD 83, P 104001-104006. http://dx.doi.org/10.1103/physrevd.83.104001.

[14] Criscienzo, R. D., Nadalini, M., Vanzo, L., Zerbini, S., Zoccatelli, G., (2007). On the Hawking radiation as tunneling for a class of dynamical black holes. Physics Letter B 657, P 107-111. http://dx.doi.org/10.1016/i.physletb.2007.10.005.

[15]Jiang, K. X., Feng, T., Peng, D. T., (2009).Hawking radiation of apparent horizon in a FRW universe as tunneling beyond semiclassical approximation. International Journal of Theoretical Physics 48, P 2112-2121. http://dx.doi.org/10.1007/s10773-009-9988-y.

[16] Angheben, M., Nadalini, M., Vanzo, L., Zerbini, S. (2005). Hawking radiation as tunneling for extremal and rotating black hole. Journal of High Energy Physics 05, 014. http://dx.doi.org/10.1088/11266708/2005/05/014.

[17] Bo, L., Wen-Biao, L., (2010). Negative temperature of inner horizon and Planck absolute entropy of a Kerr-Newman black hole. Communication Theoretical Physics 53, P 83-86. http://dx.doi.org/10.1088/0253-6102/53/1/19.

[18] Misra, R., Mahanta, C. R., (2013a). Corrected Hawking Temperature of Warped AdS3 rotating black hole by using Tunneling method. Astrophysics and Space science, Vol. No. 344 Issue I P 63-67.

[19]Mahanta, C. R., Misra, R., (2013b).Corrected Hawking Temperature of $(2+1)$ dimensional BTZ (Banados-Teitelboim-Zanelli) rotating black holeby using Tunneling method.Astrophysics and Space science, Vol. No. 348 Issue 2 437-440.

[20]Arzano, M., Medved, A. J. M., Vagenas, E. C., (2005). Hawking radiation as tunneling through the quantum horizon. Journal of High Energy Physics 09, 037. http://dx.doi.org/10.1088/11266708/2005/09/037.

[21] Medved, A. J. M., Vagenas, E. C., (2005). On hawking radiation as tunneling with back reaction. Modern Physics Letters A 20, P 2449 2454. http://dx.doi.org/10.1142/s021773230501861x.

[22]Akhmedov, E. T., Akhmedova, V., Singleton, D., (2006). Hawking temperature in the tunneling picture. Physics LetterB 642, P 124-128. http://dx.doi.org/10.1016/j.physletb.2006.09.028.

[23] Modak, S. K., (2009). Corrected entropy of BTZblack hole in tunneling approach. Physics LetterB 671, P 167-173. http://dx.doi.org/10.1016/j.physletb.2008.11.043.

[24]Banerjee, R., Modak, S. K., 2009b. Exact differential and corrected area law for stationary black holes in tunneling method. Journal of High Energy Physics 05, 063. 
[25]Unruh, W. G., 1981. Experimental Black-Hole Evaporation? Physical Review Letters 46, P 1351-1353. http://dx.doi.org/10.1103/PhysRevLett.46.1351.

[26] Unruh, W. G., 1995. Sonic analogue of black holes and the effects of high frequencies on black hole evaporation. Physical Review D 51, P 2827-2838. http://dx.doi.org/10.1103/physrevd.51.2827.

[27]Visser, M., (1998). Acoustic black holes: Horizons, ergospheres, and hawking radiation. Classicaland Quantum Gravity 15, P 1767-1791. http://dx.doi.org/10.1088/0264-9381/15/6/024.

[28] Visser, M., (1993). Acoustic propagation in fluids: An unexpected example of Lorentzian geometry. [arxiv gr-qc/9311028]

[29]Visser, M., 1999. Acoustic black holes. [arxiv gr-qc/9311028]

[30]Novello, M., Visser, M., Volovik, G., (2002). Artificial black holes. River Edge, USA: world scientific 391.

[31]Barcelo, C., Leberati, S., Visser, M., (2005). Analogue Gravity. Living reviews in relativity 8, 12. http://dx.doi.org/10.12942/lrr-2005-12.

[32]Berti, E., Cardoso, V., Lemos, J. P. S., (2004).Quasinormal modes and classical wave propagation in analogue black holes. Physical Review D 70, P 124006-124024.http://dx.doi.org/10.1103/physrevd.70.124006.

[33]Lepe, S., Saavedra, J., (2005).Quasinormal modes, superradiance and area spectrum for $(2+1)$ acoustic black holes. Physics Letter B 617 P 174-181 [arviv:grqc/0410074]http://dx.doi.org/10.1016/i.physletb.2005.05.021.

[34]Berti, E., (2005). Black Hole in a bathtub. Journal of Physics: conference series 8, P 101-105.http://dx.doi.org/10.1088/1742-6596/8/1/013.

[35]Kim, W., Park, Y. J., Son, E. J., (2006). Statistical entropy and superrdiance in (2+1)-dimensional acoustic black holes. Journal of the Korean Physical society, Vol. 49, P15-20.

[36]Bercelo, C., Liberati, S., Sonego, S., Visser, M., (2006). Quasi-particle creation by analogue black hole. Classical and Quantum Gravity 23, P 5341-5366. http://dx.doi.org/10.1088/0264-9381/23/17/014.

[37]Carusotto, L., Fagnocchi, S., Recati, A., Balbinot, R., Fabbri, A., (2008).Numerical observation of Hawking radiation from acoustic black holes in atomic Bose-Einstein condensates. New Journal of Physics 10, P 103001 103015. http://dx.doi.org/10.1088/13672630/10/10/103001.

[38] Kim, W., Son, E.J., Yoon, Myungseok., (2008). Thermodynamics of $(2+1)$ dimensional acoustic black hole based on the generalized uncertainty principle. [arxiv: 0801. 1439]

[39]Lombardo, F. C., Turiaci, G. J., (2012).Decoherence and loss of entanglement in acoustic black holes. Physical Review Letters108, P 261301-261305. http://dx.doi.org/10.1103/PhysRevLett.108.261301.

[40]Lemos, J. P. S., (2013). Rotating analogue black holes: Quasinorma modes and tails, superresonance, and sonic bombs and plants in the draining bathtub acoustic hole. [arxiv: 1312.7176]

[41]Basak, S., Majumdar, P., (2003).Superresonance from a rotating acoustic black hole. Classical and Quantum Gravity 20, P 3907-3914. http://dx.doi.org/10.1088/0264-9381/20/18/304.

[42] Banados, M., Teitelboim, C., Zanelli, J., (1992).Black hole in threedimensional spacetime. Physical Review Letters 69, P 1849-1851. http://dx.doi.org/10.1103/physrevlett.69.1849. 